\title{
Chemical composition of the essential oil from Calamintha nepeta (L.) Savi plants growing in the flora of Azerbaijan
}

\author{
Sevil A. Zeynalova \\ Institute of Botany, Azerbaijan National Academy of Sciences, \\ Badamdar 40, Baku, AZ1004, Azerbaijan
}

Abstract: In this work, we investigated the chemical constituents of the essential oil extracted from the aerial parts of Calamintha nepeta (L.) Savi (Laminaceae Lindl.). The aerial parts of $C$. nepeta collected from in flowering time from the village Tengalti and the near of Velvelechay river of Quba region. The essential oil was obtained ant by hydrodistillation. The amounts of the essential oil extracted from the above-ground portion of were $0.8-1.2 \%$. The color of oil was yellow with pleasant mint-like smell. Essential oil was analyzed for their chemical composition by gas chromatography (GC) and gas chromatography-mass spectrometry (GC-MS). In all, 78 compounds were identified, the major components were: Thymol (19.81\%), Cyclopropane, 1,1-diethyl- (19.77\%), Cyclohexanone, 3-vinyl3-methyl- (18.66\%), D-Limonene (7.45\%), Caryophyllene $(6.16 \%)$, the minor components were: beta.-Pinene (1.46\%), 3-Octanol (1.85\%), Terpinen-4-ol (1.43\%), alpha.-Terpineol (1.6\%), 1,4,7,-Cycloundecatriene, 1,5,9,9- tetramethyl-, Z,Z,Z- (3.10\%), Germacrene D (1.18\%), Caryophyllene oxide $(2.41 \%), 12$-Oxabicyclo [9.1.0]dodeca-3,7-diene, 1,5,5,8-tetramethyl-, [1R$\left.\left(1 \mathrm{R}^{*}, 3 \mathrm{E}, 7 \mathrm{E}, 11 \mathrm{R}^{*}\right)\right](1.32 \%)$. The components which the percentage of content less than one -alpha-Pinene (0.75\%), Cyclohexanol, 1-methyl-4-(1-methylethenyl)-, cis- $(0.85 \%)$, beta.-Ocimene $(0.74 \%)$, O-Cymen5-OL $\quad(0.76 \%), \quad$ 2-Dimethylamino-3-methylpyridine $(0.77 \%), 3,4$-Dimethoxytoluene $(0.54 \%), 10,10$-Dimethyl-2,6-Dimethylenebicyclo[7.2.0] undecan-5.beta.ol $(0.49 \%)$, Alloaromadendrene $(0.35 \%)$, trans-Z-. alpha.-Bisabolene epoxide $(0.38 \%)$ et al. This is the first report on the chemical compounds of the essential oil of this species growing in the territory of Azerbaijan.

Key Words: Calamintha nepeta, Lamiaceae, essential oil, GC and GC-MS, compounds.

Accepted for publication: 10 December 2018

1'E-mail: zsevil@mail.ru

\section{INTRODUCTION}

Calamintha nepeta (L.) Savi (Calamintha officinalis (Moenc) [Czerepanov, 1995] is a plant species belonging to the family Lamiaceae Lindl. The genus Calamintha Mill. is distributed in Europe, Eastern Mediterranean region, Central Asia, North Africa and America. There are more than 7 species in the world flora, of which 4 species are found in the Caucasus and up 3 species in Azerbaidjan [Askerov, 2008, 2011; Flora Azerbaydjana, 1957].

There are a lot of literature information relating to research biological activities and the essential oil extracted from the Calamintha species [Kitic et al., 2005; Alan et al., 2010; Septi et al., 2011; Regina et al., 2012]. Aforementioned species contain bioactive materials like essential oils, flavonoids, vitamin C, saponins, triterpenoide, tanins, and more [Rastitelnie resursi, 1991, 1996, 2011]. Because of their pleasant mint-like smell, many Calamintha species are used as spices in various culinary recipes. They are used in folk medicine like mints, mainly as stimulant, digestive, tonic, antiseptic etc. [Rastitelnie resursi, 1991; Chevallier, 2001]. These plants are used as antispasmodic, diaphoretic, diuretics, carminatives expectorant and for strengthening central nervous system [Grieve, 1982; Baytop, 1999; Bown, 2001; Nostro et al., 2002; Nostro et al., 2004; Burzo et al., 2006; Formisano et al., 2007; Alan et al, 2011; Mancini et al., 2013; Araniti, Grana et al., 2013; Colombo et al., 2013; Araniti, Grana et al., 2013]. Investigations showed that leaves and flowers of Calamintha species are effective as an antiseptic, antispasmodic and tonic [Small, 2006; Branković, 2009; Demirci et al., 2011; Ćavar, et al., 2013; Rastitelnie resursi, 1991, 1996, 2011], as well as antimicrobial and antispasmodic activities of their essential oils [Branković, 2009; Sarac, Ugur, 2009; Demirci et al., 2011]. The oils of some species exert significant sedating and antipyretic activities [De Ortiz Urbina et al., 1989; Rastitelnie resursi, 1991; Formisano, 2007]. Calamintha essential oils are also used for stomach, throat aches and kidney disorders [Rastitelnie resursi, 1991; Viney, 1994; Alan, et al., 2011; Ćavar, et al., 2013]. Calamintha species also have horticultural uses [Rastitelnie resursi, 1991; Baytop, 1999].

In order to investigate essential oils from local wild plants, one of the Calamintha species, C. nepeta (L.) Savi, was screened. Based on results, it was revealed 
that $C$. nepeta is widely used in traditional medicine and as a spice in cousin.

The species contains such bioactive compounds as essential oil, flavonoids, vitamin $\mathrm{C}$, saponins, triterpenoids, iridoides and etc. C. nepeta essential oil is used in cooking as an aromatic herb and also to improve the flavor and fragrance of several pharmaceutical products. Lately, the essential oil of $C$. nepeta used for its diaphoretic, expectorant, and flavouring properties has been reported for its preservative properties in culture medium and in cetomacrogol cream [Moattar et al., 2018; Morteza-Semani et al., 2007; Mancini et al., 2013; Marongiu et al., 2010; Eftekharinasab et al., 2012; Nostro et al., 2002; Nostro et al., 2004; Riela et al., 2008; Rastitelnie resursi, 1991, 1996, 2011]. The microbial activity of the Essential oil of Calamintha nepeta was screened against Aspergillus niger, Escherichia coli, Staphylococcus aureus, Salmonella enteritidis, Bacillus subtilis and Pseudomonas aeruginosa. It was found that all mentioned microorganisms were sensitive to the oil [Kitic et al., 2005; Rastitelnie resursi, 1991].

The aim of this study was to determine the quantity content of component of essential oil of $C$. nepeta.

\section{MATERIAL AND METHODS}

During our study, aerial parts of Calamintha grandiflora (L.) Moench were collected in 10th of July 2017 from the village Tengalti and the near of Velvelechay river of Quba region.

The essential oil of $C$. nepeta has been obtained by hydrodistillation [Qinzberg, 1932]. The composition of the essential oil obtained from the dried parts C.nepeta was analyzed by gas chromatography (GC) and gas chromatography-mass spectrometry (GC-MS).

GC and GC-MS conditions. The oils were analyzed by capillary GC and GC/MS using a Agilent GC-MSD system (Agilent Technologies Inc., Santa Clara,CA).

Gas Chromatography (GC) analysis was conducted using a Shimadzu GC-17A with a capillary CP-SIL $8 \mathrm{CB}$ column $(15 \mathrm{~m}$ x $0.25-0.39 \mathrm{~nm})$. Nitrogen was used as the carrier gas at the constant flow rate of $5 \mathrm{ml} /$ min. The oven temperature was kept at $60^{\circ} \mathrm{C}$ for $2 \mathrm{~min}$, then programmed to $250^{\circ} \mathrm{C}$ at a rate of $6^{\circ} \mathrm{C} / \mathrm{min}$ for 5 $\mathrm{min}$, and then kept to $280^{\circ} \mathrm{C}$ of $15^{\circ} \mathrm{C} / \mathrm{min}$ for $6 \mathrm{~min}$. The injector and detector (FID) temperatures were kept at $280^{\circ} \mathrm{C}$ and $300^{\circ} \mathrm{C}$, respectively. Gas ChromatographyMass Spectroscopy (GC-MS) analysis was carried out using an Agilent 6890N Network CG system combined with an Agilent 5975 inert Mass Selective Detector. The GC conditions were: capillary column Agilent
19091S-433 HP-5MS 5\% Phenyl Methyl Siloxane; nominal length $30.0 \mathrm{~m}$, nominal diameter $250.00 \mathrm{um}$, nominal film thickness $0.25 \mathrm{um}$; oven temperature was kept at $70^{\circ} \mathrm{C}$ for $2 \mathrm{~min}$, then programmed to $280^{\circ} \mathrm{C}$ at a rate of $5^{\circ} \mathrm{C} / \mathrm{min}$, and then held for $6 \mathrm{~min}$. Helium was used as a carrier gas; nominal unit pressure $7.64 \mathrm{psi}$; average velocity $36 \mathrm{~cm} / \mathrm{sec}$; initial flow $1.0 \mathrm{~mL} / \mathrm{min}$; split ratio 40:1; injected volume, $0.50 \mu \mathrm{L}$. Identification of the components was done by comparison of the mass spectral data using the Wiley and NIST 11.L electronic libraries. The percentages of the components were calculated from the GC peak areas, using the normalization method.

\section{RESULTS AND DISCUSSION}

C. nepeta refers to perennial plant which grows up 20$50 \mathrm{sm}$. Leaves on petioles, broadly ovate, pubescent on both sides. Inflorescence - the whorls 3-7-flowered. Corolla is pink. Fruit - nut spherical. Flowering time in July-November, fruiting - from August to November. It is xeromezophyte. Grows as groups in lighted forests and part of the riverine forest; found from the lowlands to the middle mountain belts.

The amounts of the essential oil extracted from the above-ground portion of $C$. nepeta were $0.8-1.2 \%$. The color of oil was yellow of with pleasant mint-like smell. The composition of the essential oil obtained from the dried parts of C. nepeta was analyzed by GC and GCMS. In all, 78 compounds were identified. Constituents of the essential oils of $C$. nepeta are shown in Table. As can be seen from the table, Thymol (19.81\%), Cyclopropane, 1,1-diethyl- (19.77\%), Cyclohexanone, 3-vinyl-3-methyl- (18.66\%), D-Limonene (7.45\%), Caryophyllene $(6.16 \%)$ are the major components and 1,4,7,-Cycloundecatriene, 1,5,9,9- tetramethyl-, Z,Z,Z- (3.10\%), Caryophyllene oxide (2.41\%), 3-Octanol $(1.85 \%)$, alpha.-Terpineol $(1.60 \%)$, beta.-Pinene $(1.46 \%)$, Terpinen-4-ol (1.43\%) are minor components of the essential oil of Calamintha nepeta. It is followed by the components which the percentage of content $<1$ : alpha.-Pinene (0.75\%), Cyclohexanol, 1-methyl-4-(1methylethenyl)-, cis- $(0.85 \%)$, beta.-Ocimene $(0.74 \%)$, O-Cymen-5-OL (0.76\%), 2-Dimethylamino-3-methylpyridine $(0.77 \%), 3,4$-Dimethoxytoluene $(0.54 \%)$, 10,10-Dimethyl-2,6-dimethylenebicyclo[7.2.0]undecan-5.beta.-ol (0.49\%), Alloaromadendrene (0.35\%), trans-Z-.alpha.-Bisabolene epoxide $(0.38 \%)$ etc. 


\section{PLANT \& FUNGAL RESEARCH}

Table. The composition of the essential oil of Calamintha nepeta.

\begin{tabular}{|c|c|c|c|}
\hline $\begin{array}{l}\text { Peak } \\
\text { No. }\end{array}$ & $\begin{array}{l}\text { Retentio } \\
\text { n time }\end{array}$ & Compounds & $\begin{array}{c}\text { Area } \\
(\%)\end{array}$ \\
\hline 1 & 2 & 3 & 4 \\
\hline 1 & 6.404 & alpha.-Pinene & 0.75 \\
\hline 2 & 7.040 & beta.-Pinene & 1.46 \\
\hline 3 & 7.269 & 3-Octanol & 1.85 \\
\hline 4 & 7.534 & $(+)$-4-Carene & 0.20 \\
\hline 5 & 7.710 & D-Limonene & 7.45 \\
\hline 6 & 8.057 & gamma.-Terpinene & 0.461 \\
\hline 7 & 8.234 & Cyclohexanol, 1-methyl-4-(1-methylethenyl)-, cis- & 0.85 \\
\hline 8 & 8.381 & 2-Carene & 0.27 \\
\hline 9 & 8.528 & 1,6-Octadien-3-ol, 3,7-dimethyl- & 0.41 \\
\hline 10 & 8.598 & beta.-Ocimene & 0.74 \\
\hline 11 & 8.840 & 2,4,6-Octatriene, 2,6-dimethyl-, (E,Z)- & 0.24 \\
\hline 12 & 8.957 & 1,4-Benzenediol, 2,5-dimethyl- & 0.35 \\
\hline 13 & 9.216 & l-Menthone & 0.15 \\
\hline 14 & 9.310 & Cyclohexanone, 5-methyl-2-(1-methylethyl)-, trans- & 0.13 \\
\hline 15 & 9.492 & Terpinen-4-ol & 1.43 \\
\hline 16 & 9.645 & alpha.-Terpineol & 1.60 \\
\hline 17 & 9.869 & L-.alpha.-Terpineol & 0.89 \\
\hline 18 & 10.522 & Cyclopropane, 1,1-diethyl- & 19.77 \\
\hline 19 & 10.857 & Thymol & 19.81 \\
\hline 20 & 11.075 & O-Cymen-5-OL & 0.76 \\
\hline 21 & 11.169 & 2-Methoxy-4-vinylphenol & 0.22 \\
\hline 22 & 11.239 & Phenol, m-tert-butyl- & 0.14 \\
\hline 23 & 11.628 & Cyclohexanone, 3-vinyl-3-methyl- & 18.66 \\
\hline 24 & 11.739 & (+)-3-Carene, 2-(acetylmethyl)- & 0.54 \\
\hline 25 & 11.798 & 2-Dimethylamino-3-methylpyridine & 0.77 \\
\hline 26 & 11.986 & Caryophyllene & 6.16 \\
\hline 27 & 12.133 & 1,6-Cyclodecadiene, 1-methyl-5-met hylene-8-(1-methylethyl)-, [S-(E,E)] & 0.15 \\
\hline 28 & 12.257 & 1,4,7,-Cycloundecatriene, 1,5,9,9- tetramethyl-, Z,Z,Z- & 3.10 \\
\hline 29 & 12.439 & Germacrene D & 1.18 \\
\hline 30 & 12.533 & gamma.-Muurolene & 0.06 \\
\hline 31 & 12.598 & beta.-copaene & 0.10 \\
\hline 32 & 12.651 & Benzene, 1-methyl-3-[(2-methylpropyl)thio]- & 0.22 \\
\hline 33 & 12.739 & 2-Butanone, 4-(4-hydroxy-3-methoxyphenyl)- & 0.10 \\
\hline 34 & 12.810 & Benzene, 1,2,3-trimethoxy-5-(2-propenyl)- & 0.06 \\
\hline 35 & 12.939 & 3,4-Dimethoxytoluene & 0.54 \\
\hline 36 & 13.016 & Caparratriene & 0.12 \\
\hline 37 & 13.122 & 6-Cyano-5-methoxyquinoline & 0.21 \\
\hline 38 & 13.210 & 1-Methyl-6-(3-methylbuta-1,3-dienyl)-7-oxabicyclo[4.1.0]heptane & 0.21 \\
\hline 39 & 13.316 & Caryophyllene oxide & 2.41 \\
\hline 40 & 13.428 & Humulene & 0.17 \\
\hline 41 & 13.539 & $\begin{array}{l}\text { 12-Oxabicyclo[9.1.0]dodeca-3,7-diene, 1,5,5,8-tetramethyl-, [1R-(1R* } \\
\text {,3E,7E,11R*)]- }\end{array}$ & 1.32 \\
\hline 42 & 13.751 & 10,10-Dimethyl-2,6-dimethylenebicyclo[7.2.0]undecan-5.beta.-ol & 0.49 \\
\hline 43 & 13.898 & Alloaromadendrene & 0.35 \\
\hline 44 & 14.039 & trans-Z-.alpha.-Bisabolene epoxide & 0.38 \\
\hline 45 & 14.263 & $\begin{array}{l}\text { Naphthalene, 1,2,3,4-tetrahydro-1,6-dimethyl-4-(1-methylethyl)-, (1S- } \\
\text { cis)- }\end{array}$ & 0.16 \\
\hline 46 & 14.439 & $\begin{array}{l}\text { Cyclohexane, 1,5-diethenyl-3-methy l-2-methylene-, (1.alpha.,3.alpha. } \\
\text {,5.alpha.)- }\end{array}$ & 0.10 \\
\hline
\end{tabular}


ZEYNALOVA: ESSENTIAL OIL OF CALAMINTHA NEPETA

\begin{tabular}{|c|c|c|c|}
\hline 1 & 2 & 3 & 4 \\
\hline 47 & 14.774 & Cedren-13-ol, 8- & 0.04 \\
\hline 48 & 15.269 & $\begin{array}{l}\text { 6.beta.Bicyclo[4.3.0]nonane, 5.beta.-iodomethyl-1.beta.-isopropenyl- } \\
\text { 4.alpha.,5.alpha.-dimethyl-, }\end{array}$ & 0.06 \\
\hline 49 & 15.669 & Isoaromadendrene epoxide & 0.03 \\
\hline 50 & 15.839 & 2-Pentadecanone, 6,10,14-trimethyl & 0.06 \\
\hline 51 & 15.157 & Phthalic acid, isobutyl octyleste & 0.08 \\
\hline 52 & 16.492 & 9-Isopropyl-1-methyl-2-methylene-5-oxatricyclo[5.4.0.0(3,8)]undecane & 0.11 \\
\hline 53 & 16.921 & trans-Geranylgeraniol & 0.03 \\
\hline 54 & 17.092 & Hexadecanoic acid, methyl ester & 0.05 \\
\hline 55 & 17.604 & Dibutyl phthalate & 0.05 \\
\hline 56 & 18.015 & Aromadendrene oxide-(2) & 0.02 \\
\hline 57 & 19.862 & Heneicosane & 0.03 \\
\hline 58 & 20.004 & Phytol & 0.19 \\
\hline 59 & 20.392 & $\begin{array}{l}\text { Furazano[3,4-b]pyrazine-5,6-diamine, N-isopropyl-N'-(3,4- } \\
\text { dimethylphenyl)- }\end{array}$ & 0.06 \\
\hline 60 & 21.192 & Imidazole, 4-amino-5-ethoxycarbonyl- & 0.06 \\
\hline 61 & 21.474 & Biphenyl, 4,4'-bis(trimethylsilyl) & 0.09 \\
\hline 62 & 21.674 & Imidazole, 4-amino-5-ethoxycarbonyl- & 0.06 \\
\hline 63 & 22.050 & 2(1H)-Pyridinethione, 1-ethyl-6-methyl- & 0.03 \\
\hline 64 & 22.797 & 3,5-Dihydroxybenzamide & 0.04 \\
\hline 65 & 22.909 & Heptadecane & 0.03 \\
\hline 66 & 23.456 & Cyclodisilazane, 2,2,4,4-tetramethyl-1,3-diphenyl- & 0.10 \\
\hline 67 & 23.539 & $\begin{array}{l}\text { 2H-3,7-Methanoazacycloundecino[5, 4-b]indole, 7-ethyl-1,4,5,6,7,8,9,10- } \\
\text { octahydro-11-methoxy-, (-)- }\end{array}$ & 0.03 \\
\hline 68 & 24.003 & 1-Methyl-4-ethylaminocytosine & 0.03 \\
\hline 69 & 24.339 & Tetracosane & 0.03 \\
\hline 70 & 25.468 & 4,4-Bis(1,1-dimethylethyl)-3'-methoxy-1.1'-biphenyl-2-ol & 0.03 \\
\hline 71 & 25.586 & Benzene, 2-methoxy-1,3,4-trimethyl & 0.06 \\
\hline 72 & 25.715 & 3-(3-Hydroxy-4-methyl-phenyl)-3,4,4-trimethyl-cyclopentanone & 0.07 \\
\hline 73 & 26.091 & Phthalic acid, di(2-propylpentyl) ester & 0.06 \\
\hline 74 & 27.027 & Heneicosane & 0.03 \\
\hline 75 & 28.291 & Eicosane & 0.04 \\
\hline 76 & 29.444 & Decanedioic acid, bis(2-ethylhexyl) ester & 0.02 \\
\hline 77 & 30.044 & $\begin{array}{l}\text { 2-[[3-(3,5-dimethyl-pyrazol-1-yl)- [1,2,4]triazol-4-ylimino]-methyl]- } \\
\text { phenol }\end{array}$ & 0.03 \\
\hline 78 & 30.673 & Eicosane & 0.03 \\
\hline
\end{tabular}

\section{CONCLUSIONS}

In the course of this study, it was revealed that during the flowering phase in the aerial part of C. nepeta (L.) Savi, collected from the village Tengalti and the near of Velvelechay river of Quba region the content of essential oil varies from $0.8 \%$ to $1.2 \%$.

According to our results, essential oil components of Calamintha were conditionally divided into three groups (A, B, C). A groupincludes the leading components Thymol (19.81\%), Cyclopropane, 1,1-diethyl- (19.77\%),
Cyclohexanone, 3-vinyl-3-methyl- (18.66\%), D-Limonene $(7,45 \%)$, Caryophyllene $(6.16 \%)$. B components which percentage of $>1$ : beta.-Pinene (1.46\%), 3-Octanol $(1.85 \%)$, Terpinen-4-ol (1.43\%), alpha.-Terpineol (1.6\%), 1,4,7,-Cycloundecatriene, 1,5,9,9- tetramethyl-, Z,Z,Z- (3.10\%), Germacrene D (1.18\%), Caryophyllene oxide (2.41\%), 12-Oxabicyclo[9.1.0]dodeca-3,7-diene, 1,5,5,8-tetramethyl-, [1R-(1R*,3E,7E,11R*)]- (1.32\%) and $\mathrm{C}-<1$ : alpha.-Pinene $(0.75 \%)$, Cyclohexanol, 1-methyl-4-(1-methylethenyl)-, cis- $(0.85 \%)$, beta.- 
PLANT \& FUNGAL RESEARCH 
Ocimene (0.74\%), O-Cymen-5-OL (0.76\%), 2-Dimethylamino-3-methylpyridine $\quad(0.77 \%), \quad 3,4-\mathrm{Di}-$ methoxytoluene $(0.54 \%), \quad 10,10$-Dimethyl-2,6-dimethylenebicyclo[7.2.0]undecan-5.beta.-ol (0.49\%), Alloaromadendrene (0.35\%), trans-Z-.alpha.-Bisabolene epoxide $(0.38 \%)$ et al.

\section{REFERENCES}

Alan S., Kürkçüoğlu M., Baser K.H.C. (2010) Composition of the essential oils of Calamintha sylvatica Bromf. subsp. sylvatica and Calamintha sylvatica Bromf. subsp. ascendens (Jordan) P.W. Ball. J Essent Oil Res. 22(4): 325-327.

Alan S., Kürkçüoglu M., Hüsnü K., Baser K. (2011) Composition of essential oils of Calamintha nepeta (L.) Savi subsp. nepeta and Calamintha nepeta (L.) Savi subsp. glandulosa (Req.) P.W. Ball. Asian J. Chem. 23: 2357-2360.

Araniti F., Grana E., Reigosa M.J., Sanchez-Moreiras A.M., Abenovoli M.R. (2013) Individual and joint activity of terpenoids, isolated from Calamintha nepeta extract, on Arabidopsis thaliana. Nat Prod Res.; 27(24): 2297-303. doi: 10.1080/14786419.2013.827193.

Araniti F., Lupini A., Sorgonà A., Statti G.A., Abenavoli M.R. (2013) Phytotoxic activity of foliar volatiles and essential oils of Calamintha nepeta (L.) Savi. Nat Prod Res. 27(18):1651-6. doi: 10.1080/14786419.2012.746337.

Askerov A.M. (2008) Higher Plants of Azerbaijan. The outline of Azerbaijan Flora. 3: 240. (In Azerbaijani)

Osgərov A.M. Azərbaycan florasının konspekti. Bak1: Elm. 2011, 202 s. (In Azerbaijani)

Baytop T. (1999) Therapy with Medicinal Plants in Turkey (Past and Present), 2nd ed., Nobel Tip Kitabevi: Istanbul, Turkey, $371 \mathrm{p}$.

Bown D. (2001) The Herb Society of America-New Encyclopedia of Herbs \& Their Uses, Dorling Kindersley: New York, NY, USA, 448 p.

Branković S.V., Kitić D.V., Radenković M.M., Veljković S.M., Golubović T.D. (2009) Calcium blocking activity as a mechanism of the spasmolytic effect of the essential oil of Calamintha glandulosa Šilić on the isolated rat ileum. Gen. Physiol. Biophys. 28; 174-178.

Burzo I., Mihaescu D., Dobrescu A., Ambăruş S., Fălticeanu M., Bădulescu L. (2006) Contribution to the knowledge of the composition of the essential oils from five Calamintha species cultivated in Romania. Alexandru Ioan Cuza University of Iaşi,
«Al. I. Cuza» din Iasi 52: 39-42.

Colombo R.P., Martínez A., Fernández di Pardo A., Fernández Bidondo L., van Baren C., di Leo Lira P., Godeas A.M. (2013) Differential effects of two strains of Rhizophagus intraradices on dry biomass and essential oil yield and composition in Calamintha nepeta. Rev Argent Microbiol. 45(2): 114-8.

Ćavar S., Vidić D., Maksimović M. (2013) Volatile constituents, phenolic compounds, and antioxidant activity of Calamintha glandulosa (Req.) Bentham. $J$. Sci. Food Agric. 93: 1758-1764.

Chevallier A. (2001) Encyclopedia of Medicinal Plants, Dorling Kindersley: London, UK, 211-212 p.

Czerepanov S.K. (1995) Vascular plants of Russia and adjacent states (the former USSR) Cambridge. $516 \mathrm{p}$.

Demirci B., Temel H.E., Portakal T., Kırmızıbekmez H., Demirci F., Başer K.H.C. (2011) Inhibitory effect of Calamintha nepeta subsp. glandulosa essential oil on lipoxygenase. Turk. J. Biochem. 36: 290-295.

De Ortiz Urbina A.V., Martín M.L., Montero M.J., Morán A., San Román L. (1989) Sedating and antipyretic activity of essential oil of Calamintha sylvatica subsp. ascendens. J. Ethnopharmacol. 25, 165-171.

Eftekharinasab N., Zarei D., Paidar S., Moghadam M. J., Kahrizi D., Khanahmadi M., Chenari P. (2012) Identification of wild medicinal plant in Dalahoo mountain and their used indigenous knowledge (Kermanshah, Iran). Annals of Biological Research, 3(7): 3234-3239.

Formisano C., Rigano D., Napolitano F., Senatore F., Apostolides A.N., Piozzi F., Rosselli S. (2007) Volatile constituents of Calamintha origanifolia Boiss. growing wild in Lebanon. Nat. Prod. Commun. 2: 1253-1256.

Grieve M. (1982) A Modern Herbal, Penguin Books, Great Britain, 807-808.

Karousou Regina, Hanlidou Effie, Lazari Diamanto (2012) Essential oil diversity of three Calamintha species from Greece. Chemistry \& Biodiversity. 9(7): 1364-1371. doi: 10.1002/cbdv.201100262

Katayoun Morteza-Semani \& Mohammad Akbarzadeh. (2007) The Essential Oil Composition of Calamintha officinalis Moench from Iran. J Essent Oil Bear $P l, 10(6)$ : 494-498.

Kitic D., Palic R., Strojanović G., Randjelovic V. (2005) Chemical Composition and Microbial Activity of the Essential Oil of Calamintha nepeta (L.) Savi ssp. Nepeta var. subisodonda (Borb.) Hayek from Serbia. 
$J$ Essent Oil Res, 17(6): 701-703. doi:10.1080/10412905.2005.9699036

Mancini E., De Martino L., Malova H., De Feo V. (2013) Chemical composition and biological activities of the essential oil from Calamintha nepeta plants from the wild in southern Italy. Nat Prod Commun; 8(1):139-42.

Marongiu B., Piras A., Porcedda S., Falconieri D., Maxia A., Gonçalves M.J., Cavaleiro C., Salgueiro L. (2010) Chemical composition and biological assays of essential oils of Calamintha nepeta (L.) Savi subsp. nepeta (Lamiaceae). Nat Prod Res, 24(18):173442. doi: 10.1080/14786410903108944.

Moattar F. Sh., Sariri R., Giahi M., Yaghmaee P. (2018) Essential Oil Composition and Antioxidant Activity of Calamintha officinalis Moench. J. Appl Biotechnol Rep. 2018, 5(2): 55-58. doi:10.29252/ jabr.05.02.03.

Nostro A., M.A. Cannatelli, I. Morelli, P.L. Cioni, A. Bader A. Marino 1 and V. Alonzo (2002) Preservative properties of Calamintha officinalis essential oil with and without EDTA. J Appl Microbiol, 2002, 35: 385-389.

Nostro A., Cannatelli M.A., Morelli I., Musolino A.D., Scuderi F., Pizzimenti F., Alonzo V. (2004) Efficiency of Calamintha officinalis essential oil as preservative in two topical product types. J Appl Microbiol. 97(2): 395-401. doi: 10.1111/j.1365-2672.2004.02319.x

Riela S, Bruno M, Formisano C, Rigano D, Rosselli S, Saladino ML, Senatore F. (2008) Effects of solventfree microwave extraction on the chemical composition of essential oil of Calamintha nepeta (L.) Savi compared with the conventional production method. J Sep Sci.; 31(6-7): 1110-7.

doi: $10.1002 /$ jssc. 200700425 .

Sarac M., Ugur A. (2009) The in vitro antimicrobial activities of the essential oils of some Lamiaceae species from Turkey. J. Med. Food 12: 902-907.

Septi M., Lahouel M. (2011) Chemical composition variation in essential oils of Calamintha hispidula (Boissier and Reuter) Maire, endemic in North-eastern Algeria. Planta Med; 77 - PE25

DOI: $10.1055 / \mathrm{s}-0031-1282356$

Small E. (2006) Culinary Herbs, NRC Research Press: Ottawa, ON, Canada, 1-236.

Viney D.E. (1994) An Illustrated Flora of North Cyprus, Koeltz Scientific Books: Koenigstein, Germany, 514-515.

Qinzberg A.S. (1932) Uproshenniy sposob opredele- niya kolichestva efirnoqo masla $\mathrm{v}$ efirinisax. Ximikofarmasevticheskaya promishlennost, 326-329. (In Russian)

Flora Azerbayjana. (1957) Sem. Labiatae. Pod redaksiyey I.I. Karjaqin. Izd. AN Azerb. SSR. 7: 364-365. (In Russian)

Rastitelnie resursi SSSR. Svetkovie rasteniya, ix ximicheskiy sostav, ispolzovanie. Sem. Hippuridaceae - Lobeliaceae.(1991) Otv.Redaktor P.D.Sokolov Sank-Peterburg: Nauka, 6: 17-18. (In Russian)

Rastitelnie resursi Rossii i sopredelnix qosudarstv (1995). Otv.Redaktor A.L.Budansev. Sank-Peterburg: Mir i semya, 9: 295. (In Russian)

Rastitelnie resursi Rossii. Dikorastushie svetkovie rasteniya, ix komponentniy sostav i biologicheskaya aktivnost (2011). Otv.Redaktor A.L. Budansev. Sank-Peterburg-Moskva: Tovarishestvo nauch. izd. $K M K$, 4: 629. (In Russian)

Teresa M.M., Tzakou O., Nostro A., Zimbalatti V., Galati E.M. (2011) Chemical composition and biological activities of Calamintha officinalis Moench essential oil. J Med Food. 14(3). doi.org/10.1089/jmf.2009.0191

Verma M., Irchhaiya R., Singh P.P., Kailasiya D., Kanaujia V. (2011) Studies on antiulcer activity of essential oil of Calamintha officinalis Moench. IJP$S R, 2(10):$ 2733-2736.

\section{Azorbaycan florasında yayılan Calamintha nepeta (L.) Savi bitkisindən alınan efir yağının kimyəvi tərkibi}

Sevil Ә. Zeynalova

Azarbaycan Milli Elmlar Akademiyasının Botanika Institutu AZ 1004, Bakl, Azerbaycan, Badamdar 40.

Təqdim olunan məqalədə Calamintha nepeta (L.) Savi (fəs. Laminaceae Lindl.) növünün yerüstü hissəsindən alınmış efir yağının kimyəvi tərkibi tədqiq edilmişdur. Bitki çiçəkləmə fazasında Quba rayonunun Təngaltı kəndinin ətrafinda Vəlvələçay çayının yaxınlığında yı ğılmışdır. Efir yağı hidrodistillyasiya metodu ilə alınmışdır. Efir yağının çıxımı $0.8-1.2 \%$ aralığında dəyişir. Alınmış efir yağı şəffaf sarı rənglı xoş nanə ətirli mayedir. Efir yağının kimyəvi tərkibi qaz xromatoqrafı (QX) və xromato-mass-spektrometriya metodları (XMS) ilə tədqiq olunmuş və 78 komponent müəyyən edilmişdur. Calamintha nepeta növünün efir yağının major komponentləri timol (19.81\%), siklopropan, 1,1-dietil - (19.77\%), sikloheksanon, 3-vinil-3-metil - 
(18.66\%), D-limonen (7.45\%), kariofillen (6.16\%) və minor komponentləri beta-pinen $(1.46 \%)$, 3-oktanol $(1.85 \%)$, terpinen-4-ol (1.43\%), alfa.-terpineol $(1.6 \%), \quad 1,4,7$, -siklondekatrien, 1,5,9,9-tetrametil,Z,Z,Z- (3.10\%), hermakren D ( $1.18 \%)$, kariofillen oksid (2.41\%), 12-oksabisiklo [9,1,0]dodeka-3,7-dien, 1,5,5,8-tetrametil-[1R- (1R*,3E,7E,11R*)] - (1.32\%) komponentləridir. Efir yağının tərkibində, həmçinin faizi vahiddən az olan komponentlər də müəyyən edilmişdir $(<1)$ : alfa-pinen $(0.75 \%)$, sikloheksanol, 1-metil-4-(metiletenil)-sis- $(0.85 \%), \quad$ beta-oksimen (0.74\%), O-simen-5-ol $(0.76 \%)$, 2-dimetilamino-33-metilpiridin $(0,77 \%)$, 3,4-dimetoksitoluol $(0.54 \%)$, 10,10-dimetil -2,6-dimetilenbisiklo [7.2.0] undekan$5 \beta$-ol $(0.49 \%)$, alloapomadendren $(0.35 \%)$, epoksidZ-alfa-bisabolen $(0.38 \%)$ və s. Azərbaycanda yayılmış Calamintha nepeta növünün efir yağının komponent tərkibi haqqinda ilk dəfə olaraq məlumat verilir.

Açar sözlor: Lamiaceae, Calamintha nepeta, efir yağl, $Q X$ va $Q X-M S$, komponentlar

\section{Состав эфирного масла Calamintha nepeta (L.) Savi из природной флоры Азербайджана}

Севиль А. Зейналова

Институт ботаники Наџиональной Академии наук Азербайджана $A Z$ 1004, Баку, Азербайджан, Бадамдарское шоссе, 40.

В представленной работе мы исследовали химический состав эфирного масла, полученного из надземных частей Calamintha nepeta (L.) Savi (Laminaceae Lindl.). Растения были собранные в период цветения в окрестностях сел. Тенгальты вблизи реки Вельвелечай Губинского района. Эфир- ное масло было получено гидродистилляцией. Количество масла, колеблется в пределах 0,8-1,2\%. Полученное эфирное масло представляет собой прозрачную жидкость желтого цвета с приятным мятным ароматом. Химический состав масла анализировали с помощью газовой хроматографии (ГХ) и газовой хромато-масс-спектрометрии (ГХ-МС). Было выявлено 78 соединений, из них основными компонентами эфирного масла Calamintha nepeta являлись-тимол $(19.81 \%)$, циклопропан, 1,1-диэтил- $(19.77 \%)$, циклогексанон, 3-винил-3-метил(18.66\%), D-лимонен (7.45\%), кариофиллен (6.16\%), и минорные компоненты: бета-пинен $(1.46 \%), 3$-октанол (1.85\%), терпинен-4-ол (1.43) \%), альфа.-терпинеол $(1.6 \%), 1,4,7$, -циклондекатриен, 1,5,9,9-тетраметил-, Z, Z, Z- (3.10\%), гермакрен D ( $1.18 \%)$, оксида кариофиллена $(2.41 \%)$, 12-оксабицикло [9,1,0]додека-3,7-диена, 1,5,5,8-тетраметил-,[1R$\left.\left(1 \mathrm{R} *, 3 \mathrm{E}, 7 \mathrm{E}, 11 \mathrm{R}^{*}\right)\right]$ - $(1.32 \%)$. Далее следуют компоненты, процентное содержание которых было меньше единицы $(<1)$, это - альфа-пинен $(0.75 \%)$, циклогексанол, 1-метил-4- (1-метилэтенил)-, цис- $(0.85 \%)$, бета-оксимен $(0.74 \%)$, O-Cymen5-OL $(0.76 \%), \quad$ 2-диметиламино-3-метилпиридин $(0.77 \%), 3,4$-диметокситолуол $(0.54 \%), 10,10$-диме-

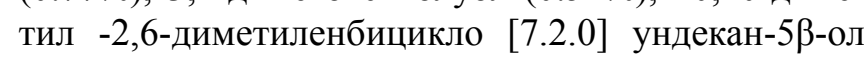
$(0.49 \%)$, аллоаромадендрен $(0.35 \%)$, эпоксид транс$\mathrm{Z}$-альфа-бисаболена $(0.38 \%)$ и другие. В данной работе впервые приводится компонентный состав эфирного масла растений Calamintha nepeta, произрастающих в Азербайджане.

Ключевье слова: Lamiaceae, Calamintha nepeta, эфирное масло, ГХ и ГХ-МС, компоненть 